\title{
NORBERT WIENER AND POTENTIAL THEORY
}

BY M. BRELOT

1. Wiener did not work in potential theory for very long (only about two years around 1924), but that was enough to bring about in this field (the so-called classical potential theory), as in many others, some fundamental contributions: a definitive form of the generalized solution of the Dirichlet problem for a continuous given boundary function, the notion of capacity for general compact sets and the famous Wiener criterion of regularity.

2. Already in 1923, he wrote with Phillips a paper [28 $]^{*}$ on Nets and the Dirichlet problem where this problem was solved for a polycubic domain then for domains with smooth boundaries by a limit process from a problem for functions on a discrete net and a mean condition. This idea of using linear equations for a preliminary problem relative to finite differences, which is now a basic tool with computers for partial differential equations was not common forty years ago; I know only the previous example of Le Roux on harmonic functions in $R^{2}$ (J. Math. Pures Appl., 1914).

3. In pure potential theory, the first fundamental paper of Wiener [24] Certain notions in potential theory in January 1924 gave and studied a precise definition of a generalized solution and the first definition of capacity for an arbitrary compact set.

For a long time, it was known that the classical Dirichlet problem does not always have a solution (case of an isolated boundary point of Zaremba, spine of Lebesgue) and there appeared more or less clearly the need to define a suitable generalized solution which always exists that would be later studied at the boundary; such a harmonic function corresponding to the given boundary function was in evidence in various methods, where further restrictions on the boundary allowed to show that it was actually a solution (Poincaré, Zaremba, Lebesgue, Bouligand, Kellogg ....). But in a clearer and more striking way than the others, Wiener introduced for a bounded open set (and for a similar "exterior" problem) a precise generalized solution, that he studied further without restrictions: it was the limit of the classical solution for an increasing sequence of regular open sets $\Omega_{n} \subset \Omega\left(U \Omega_{n}=\Omega\right)$ ("regular" means that there is always a solution for the classical Dirichlet problem) and a boundary function, given as

* The bold-faced numbers in brackets refer to the numbered references in the Bibliography of Norbert Wiener. 
the restriction on $\partial \Omega_{n}$ of any finite continuous continuation of the given function $f$ on $\partial \Omega$. This limit depends only on $\Omega$ and $f$, and is equal to the classical solution when the latter exists.

Later an easy proof appeared by using the new tool of subharmonic function and an approximation of $f$ by means of a difference $\phi_{1}-\phi_{2}$ of such functions in a larger $\Omega_{0} \supset \bar{\Omega}$, and considering the solutions in $\Omega_{n}$ for $\phi_{1}$ and $\phi_{2}$, which are increasing.

Wiener gave a rather complicated proof by considering an exterior problem relative to two compact sets and the respective boundary values 1 and 0 (at the point at infinity, the solution must tend to 0 in $R^{n}, n \geqq 3$, or must be bounded in $R^{2}$ ). Then he treated the more general case of $n$ disjoint compact sets with constant values on them and that gave an approach to the general case where the boundary was replaced by $n$ disjoint compact sets and a constant value on each one.

This difficult argument led him to consider more deeply the exterior problem with value 1 on the boundary of a given compact set $K$ in $R^{n}(n \geqq 3)$. Without the later representation of subharmonic functions by $F$. Riesz, the generalized solution was proved to be the newtonian potential of a measure $\geqq 0$ supported by $K$; the total mass was called the capacity of $K$, the basic tool of potential theory that Wiener adapted in $R^{2}$ and which has been so much used, studied and generalized. Moreover Wiener gave a sufficient condition of "regularity," broader than any previous ones. (This notion for a boundary point $x$ means that the solution tends to the given value at $x_{0}$; the regularity of every boundary point means that the open set is regular.)

4. Three months later, in [35], [36], he studied more deeply this notion of regularity, already characterized by Lebesgue and his "barrier." Wiener considered first in $R^{n}(n \geqq 3)$ the set of $C \Omega$ where the distance to $x_{0}$ lies between $\lambda^{p}$ and $\lambda^{p+1}(0<\lambda<1)$. If $\gamma_{p}$ is its capacity, the regularity is equivalent to the divergence of the series $\gamma_{p} / \lambda^{p}$. This deep property (with an adaptation in $R^{2}$ ) was difficult to prove, but became easier later with the help of advanced potential theory. This famous criterion was systematically used and later extended to the more general notion of thinness; it appeared to have a geometric character according to the geometric interpretation of capacity by Fekete-Pólya-Szegö. (However in modern axiomatics, the barrier, which has not this character, remains valid but not the Wiener criterion.)

5. A third fundamental paper [39] was published in January 1925 where Wiener compared his generalized solution with the method of 
Perron (published in Math. Z., vol. 18, where there is a quite similar and unknown paper of Remak). The method of Perron gave the classical solution (actually every time it exists) and Wiener proved that the function introduced by Perron, when considered before some complementary conditions make it the classical solution, was the generalized solution. The latter appears as the upper envelope of continuous subharmonic functions whose lim sup at the boundary minimize the given continuous data (and is a similar lower envelope). This remained valid with the further introduction of general subharmonic functions. This interpretation became the good definition and the first one a property of the generalized solution-with easy extension in the modern axiomatic theories.

The classical problem became therefore a particular case, with smaller interest.

6. Wiener could not leave aside the Dirichlet problem for a discontinuous boundary function. He was inspired by the Poissonintegral and considered the continuation of the functional defined at any $x \in \Omega$ by the solution for a given $f$ on the boundary, when $f$ becomes discontinuous, i.e., the integral of $f$ with respect to the harmonic measure. (See [39] and previously [24].) He thought of course of the identity of the Perron envelopes for large classes of $f$. He surely also would have developed this point except for a simple counterexample that the present writer discovered to be wrong 15 years later (then the identity of the suitably defined envelopes was proved to be equivalent to the summability of $f$ with respect to the harmonic measure).

In the papers I have just summarized, Wiener by mastering a difficult technique and introducing important tools initiated a new period for the Dirichlet problem and potential theory. We may be thankful for such an impulse that we may still appreciate 40 years later.

UNIVERSITY OF PARIS, PARIS, FRANCE 